\title{
Erratum to: A two-dimensional probabilistic acute human-health risk assessment of insecticide exposure after adult mosquito management
}

Jerome J. Schleier III • Paula A. Macedo •

Ryan S. Davis • Leslie M. Shama •

Robert K. D. Peterson

Published online: 17 March 2012

(C) Springer-Verlag 2012

Erratum to: Stoch Environ Res Risk Assess (2009)

\section{3:555-563}

DOI 10.1007/s00477-008-0227-5

In the published article, the values listed in Table 3 were incorrect. The corrected table is presented here.

The online version of the original article can be found under doi: 10.1007/s00477-008-0227-5.

J. J. Schleier III $(\bowtie) \cdot$ R. K. D. Peterson

Department of Land Resources and Environmental Sciences, Montana State University, 334 Leon Johnson Hall, Bozeman, MT 59717-3120, USA

e-mail: jeromes@montana.edu

P. A. Macedo

Sacramento-Yolo Mosquito and Vector Control District,

8631 Bond Road, Elk Grove, CA 95624, USA

R. S. Davis

ICM Ventures Inc., 2601 South Lemay Ave., Suite 38,

Fort Collins, CO 80525, USA

L. M. Shama

Cell Marque Corp., 6600 Sierra College Blvd., Rocklin,

CA 95677, USA 
Table 3 Acute RQ means and 95\% confidence intervals for each subgroup and chemical assessed

\begin{tabular}{|c|c|c|c|c|c|c|}
\hline Chemical & Adult male ${ }^{\mathrm{b}}$ & Adult female ${ }^{c}$ & Youth $^{\mathrm{d}}$ & Children $^{\mathrm{e}}$ & Toddlers $^{\mathrm{f}}$ & Infants ${ }^{\mathrm{g}}$ \\
\hline \multicolumn{7}{|l|}{$\mathrm{PBO}^{\mathrm{a}}$} \\
\hline Mean & 0.000009 & 0.000009 & 0.00001 & 0.00002 & 0.0002 & 0.0002 \\
\hline 95\% C.I. & 0.00001 & 0.00001 & 0.00002 & 0.00003 & 0.0003 & 0.0004 \\
\hline \multicolumn{7}{|l|}{ Phenothrin } \\
\hline Mean & 0.0001 & 0.0001 & 0.0001 & 0.0001 & 0.0002 & 0.0003 \\
\hline 95\% C.I. & 0.0001 & 0.0001 & 0.0001 & 0.0001 & 0.0003 & 0.0004 \\
\hline \multicolumn{7}{|l|}{ Permethrin } \\
\hline Mean & 0.00005 & 0.00005 & 0.00007 & 0.0001 & 0.0006 & 0.001 \\
\hline 95\% C.I. & 0.00006 & 0.00007 & 0.0001 & 0.0002 & 0.001 & 0.002 \\
\hline \multicolumn{7}{|l|}{ Resmethrin } \\
\hline Mean & 0.0001 & 0.0001 & 0.0002 & 0.0003 & 0.08 & 0.2 \\
\hline 95\% C.I. & 0.0002 & 0.0002 & 0.0003 & 0.0004 & 0.2 & 0.4 \\
\hline \multicolumn{7}{|l|}{ Malathion } \\
\hline Mean & 0.001 & 0.002 & 0.002 & 0.002 & 0.007 & 0.01 \\
\hline $95 \%$ C.I. & 0.002 & 0.002 & 0.003 & 0.003 & 0.02 & 0.02 \\
\hline \multicolumn{7}{|l|}{ Naled } \\
\hline Mean & 0.05 & 0.06 & 0.07 & 0.06 & 0.07 & 0.08 \\
\hline 95\% C.I. & 0.06 & 0.07 & 0.08 & 0.08 & 0.1 & 0.1 \\
\hline \multicolumn{7}{|l|}{ Pyrethrins } \\
\hline Mean & 0.00009 & 0.00009 & 0.0001 & 0.0002 & 0.001 & 0.002 \\
\hline 95\% C.I. & 0.0001 & 0.0001 & 0.0002 & 0.0003 & 0.003 & 0.004 \\
\hline
\end{tabular}

a Piperonyl Butoxide

b $18-65$ years of age

c 18-65 years of age

d $10-12$ years of age

e $5-6$ years of age

f $2-3$ years of age

g $0.5-1.5$ years of age 